\title{
Auto-organização e complexidade: o problema do desenvolvimento do ciclo vigília-sono
}

\author{
JOSÉ ROBERTO CASTILHO PIQUEIRA \\ - ANA AMÉLIA BENEDITO-SILVA
}

$\mathrm{O}$

S CONCEITOS DE auto-organização e complexidade têm, nos últimos anos, sido objeto de estudo nos mais diversos ramos da ciência e relacionados a uma grande diversidade de sistemas: físicos, biológicos, comportamentais, sociais, políticos, econômicos e culturais (Coveney \& Highfield, 1995).

Dentro desse panorama, interessa-nos o trabalho sobre sistemas biológicos e, principalmente, a tentativa de estabelecer relações entre conceitos da Matemática e da Física e os fenômenos biológicos, enfatizando processos e suas explanações, em vez privilegiar resultados e classificações (Gould, 1977).

Nesse sentido, um artigo sobre o assunto proposto por Piqueira (1992) apresentou uma tentativa de definir organização de um sistema por meio do conceito de medida entrópica de um sistema dinâmico. A idéia de aplicar a Teoria de Sistemas Dinâmicos a problemas biológicos sempre nos pareceu interessante, principalmente levando-se em conta a possibilidade de incluir dinâmicas, em escalas temporais diferentes, gerando as idéias de dinâmicas rápidas, lentas e intermediárias.

Embora passível de muitas críticas, o referido artigo suscitou debates proveitosos que redundaram em um segundo (Piqueira, 1994), explorando a associação das idéias de complexidade e o conceito de entropia informacional (Shannon \& Weaver, 1949). Ambos os artigos têm norteado nosso trabalho de análise de experimentos biológicos, proporcionando-nos indícios do tipo de contribuição que a Matemática pode dar à Biologia.

A tentativa de entender complexidade e auto-organização levou-nos à leitura de algumas obras clássicas da Biologia (Darwin, $1859 ; 1871$ ) as quais, dada a nossa pobre formação no assunto, geraram grande dificuldade e a consciência da necessidade de outra vida para o início de um melhor entendimento da Teoria da Evolução, fundamento básico da Biologia moderna.

Felizmente tivemos acesso a um livro que, dada sua clareza e qualidade científica, está nos ajudando bastante na tarefa que temos em mente (Dawkins, 1986) e, acreditamos, ter encontrado algumas idéias de Biologia Evolutiva que nos pareceram passíveis de formalização mediante conceitos originários da Matemática. 
É disso que pretendemos tratar. Discutiremos os pontos relativos às condições necessárias para caracterizar complexidade biológica e auto-organização indicados por Dawkins, procurando lhes dar o formalismo matemático que nos for possível.

Todas as interpretações feitas a seguir são de nossa inteira responsabilidade. Caso alguma idéia esteja incorreta, a origem não foi o texto de Dawkins, mas a nossa provável incompreensão desses conceitos.

Trataremos inicialmente da definição de complexidade, levando em conta que, na linguagem coloquial, o termo significa algo difícil de ser descrito,compreendido, montado ou concebido. Com tais idéias em mente, tentaremos definir complexidade por meio de seus atributos essenciais: decomposição, heterogeneidade, autoorganização e adequação.

Finalizaremos com um problema de cronobiologia: o estudo do estabelecimento do ritmo circadiano, no ciclo vigília-sono de bebês, utilizando a medida da entropia informacional associada aos conjuntos de dados experimentais.

\section{Complexidade e heterogeneidade}

Nesta seção procuraremos estabelecer os fundamentos teóricos da primeira condição necessária estabelecida por Dawkins, para considerar que um dado sistema é complexo. Essa condição, a heterogeneidade, parte da hipótese que um sistema é composto por partes as quais, se vistas isoladamente, não dão uma perspectiva do todo.

Aproveitaremos o exemplo de Dawkins: duas metades de um carro não formam necessariamente um carro quando justapostas, superpostas ou combinadas. Um sistema complexo é passível de decomposições sucessivas sem que nenhuma delas, isolada, traduza o comportamento do sistema.

A tentativa de colocação desse conceito, em linguagem matemática, deve ser iniciada pela suposição de que o sistema em apreço, embora complexo, seja passível de modelagem por meio de variáveis de estado as quais, uma vez conhecidas, descrevam o sistema de maneira satisfatória (Piqueira, 1994; Mainzer, 1994).

A seguir consideraremos que determinado sistema pode ser decomposto em $m$ partes, cada uma delas passível de descrição por variáveis de estado. A cada uma das partes estarão associadas $n_{i}$ variáveis de estado, com $i$ assumindo valores entre $l$ e $m$.

De acordo com esse critério de indexação, a variável de estado $x_{i}, j$ será a $j$-ésima variável de estado, relativa à $i$-ésima parte do sistema, com o índice $i$ assumindo valores de $l$ até $m$ e o índice $j$ assumindo valores de $l$ até $n_{i}$. Assim, a cada $i$-ésima parte do sistema estarão associadas $n_{i}$ variáveis de estado.

Do exposto, é possível concluir que o número total de variáveis de estado do sistema em questão será dado por: 


$$
N=\sum_{i=1}^{m} n_{i}
$$

Conforme discutido por Piqueira (1994), esse número pode expressar o grau de complexidade estrutural de um sistema, uma vez que se relaciona com a dimensão do espaço de estados necessária para descrever o sistema.

Para expressar a heterogeneidade e a emergência de sistemas qualitativamente diferentes das combinações de suas partes, consideraremos que as equações dinâmicas de uma certa $i$-ésima parte isolada são dependentes, exclusivamente, das $n_{i}$ variáveis de estado relativas a essa parte, de seus parâmetros constitutivos e do tempo (von Bertalanffy, 1968).

Matematicamente, as equações dinâmicas relativas às variáveis de estado e suas taxas de variação são expressas como dependentes das variáveis $x_{i}, j$, fixando-se $i$ e variando-se $j$, de $l$ até $n_{i}$. Além disso, entram nas equações $l_{i}$ parâmetros constitutivos $\lambda_{i, k}$ e a variável temporal $t$.

Chamando de $F_{i}$ cada uma das aplicações do tipo $F_{i}: R^{n i} \times R^{l i} \times R \rightarrow R^{n i}$, cada uma das $i$ partes do sistema será descrita por $n_{i}$ equações dinâmicas do tipo:

$$
\dot{x}_{i, j}=F_{i}\left(x_{i, 1}, x_{i, 2}, \ldots ., x_{i}, n i ; \lambda_{i, 1}, \lambda_{i, 2}, \ldots ., \lambda_{i}, l_{i}, t\right)
$$

O ponto colocado sobre $x_{i, j}$ indica a derivada temporal dessa variável e as equações, na forma apresentada em (2), relacionam-se com a evolução dinâmica das partes do sistema quando consideradas isoladamente.

O fato de o sistema ser complexo implica alterações na forma dessas equações, originárias da reorganização das partes integradas, formando o sistema como um todo. Ao tentar expressar o fato por um formalismo matemático, definiremos algumas variáveis e parâmetros de maneira vetorial com o intuito de compactar a notação.

Chamaremos de $X_{i}$ a variável vetorial de dimensão $n_{i}$, cujas componentes serão as variáveis $x_{i}, j$, com $j$ variando de $l$ até $n_{i}$. Da mesma maneira, chamaremos $\Gamma_{i}$ o vetor de dimensão $l_{i}$, relativo aos parâmetros constitutivos da $i$-ésima parte do sistema tendo, portanto, componentes $\lambda_{i, k}$, com $k$ variando de $I$ até $l_{i}$.

De acordo com a notação estabelecida, quando um sistema é complexo, composto de $\mathrm{m}$ partes associadas a variáveis de estado vetoriais $X_{i}$, com $i$ variando de $I$ até $m$, com equações dinâmicas relativas às partes do tipo (2), suas equações dinâmicas relativas ao todo são do tipo:

$$
\dot{X}_{i}=G_{i}\left(X_{1}, X_{2}, \ldots ., X_{m} ; \Gamma_{1}, \Gamma_{2}, \ldots \ldots, \Gamma_{m} ; t\right)
$$


Na expressão (3), a relação:

$G_{i}: R^{n 1} x R^{n 2} x \ldots . . \mathrm{x}^{\mathrm{nm}} \times R^{l 1} \times R^{l 2} \ldots \ldots . . R^{l m} \times R \rightarrow R^{n 1} \times R^{n 2} x \ldots . \times R^{n m}$,

que expressa a dinâmica do sistema, contém, em todos os seus componentes, todas as variáveis de estado e parâmetros constitutivos correspondentes a todas as partes.

Dessa maneira expressamos a primeira condição necessária para complexidade proposta por Dawkins: o sistema complexo é particionável, suas partes têm a dinâmica descrita por equações do tipo (2) e o sistema, como um todo, a descrita por equações do tipo (3).

A argumentação apresentada é passível de questionamentos matemáticos, biológicos e filosóficos. A seguir, relacionaremos e discutiremos aqueles percebidos.

Do ponto de vista matemático, poderiam ser feitos questionamentos sobre a continuidade e a diferenciabilidade das relações $F_{i}$ e $G_{i}$ e, até mesmo, sobre o fato de elas serem ou não funções.

Neste estudo assumimos que tal aspecto é irrelevante, pois não estamos preocupados em assegurar existência, unicidade, continuidade ou qualquer outra propriedade matemática a respeito das supostas soluções das equações. Preferimos supor que eventuais irregularidades matemáticas possam estar relacionadas com imprevisibilidades e diversidades dos comportamentos biológicos.

Uma interessante questão biológica relativa a tal abordagem refere-se a se determinada variável poderia ser indicativa do estado do sistema ou de um certo parâmetro constitutivo. A resposta a esse tipo de questão passaria pela adoção de uma certa escala temporal.

Para um engenheiro ou para um físico, o problema parece não existir. Variáveis de estado são grandezas físicas que variam rapidamente: tensão, corrente, posição, velocidade, aceleração. Parâmetros são grandezas físicas cujas variações são imperceptíveis, em uma certa escala temporal: resistência, capacitância, indutância, massa, densidade, momento de inércia.

Para o biólogo, entretanto, a escala temporal poderá não ser tão bem definida. Há processos que devem ser encarados de acordo com o objeto de estudo do ponto de vista de uma escala temporal filogenética, ontogenética ou dinâmica atual. Como conseqüência, uma mesma variável biológica pode ser considerada indicativa do estado em uma certa escala temporal e indicativa de parâmetro constitutivo em outra.

Citaremos, a título de exemplo, a questão dos ritmos circadianos estabelecidos na espécie humana. A filogênese desses ritmos poderia ser estudada sob uma perspectiva evolutiva.

Os mesmos ritmos, entretanto, poderiam ser estudados ontogeneticamente por meio de suas variações ao longo da vida. Há, ainda, a preocupação de como 
um vôo transmeridiano alteraria tais ritmos em um certo indivíduo, ao longo de uma certa viagem (Benedito-Silva, 1993).

É evidente que o mesmo processo de estabelecimento de ritmos biológicos pode ser estudado em escalas temporais completamente diferentes e, em cada caso, uma certa variável pode ser considerada indicativa de estado ou de parâmetro constitutivo. Ressaltamos, entretanto, que isso não invalida o formalismo. Tudo é uma questão de escolher corretamente, em cada caso, os elementos pertencentes ao conjunto das variáveis $X$ e os pertencentes ao conjunto dos parâmetros $\Gamma$.

A crítica de um físico seria, talvez, sobre a observabilidade do sistema, uma vez que o número de variáveis de estado poderia ser muito grande, impossibilitando a abordagem nos termos aqui propostos. A essa crítica não saberíamos responder sem usar uma pequena evasiva: talvez pudessem ser definidas variáveis de estado que englobassem outras, assim como a termodinâmica trabalha, macroscopicamente, com pressão, volume e temperatura, com o intuito de expressar o estado de movimento de uma infinidade de moléculas de um gás.

Finalmente, um filósofo diria que nossa abordagem é determinística, com o que concordaríamos. Entretanto, acrescentamos que a estocasticidade poderia ser introduzida, tanto nas equações do tipo (2) como do tipo (3), considerando os parâmetros como variáveis aleatórias, e as funções $\mathrm{F}$ e $\mathrm{G}$ como processos estocásticos (Loève, 1963).

Além disso, há a estocasticidade natural originária da sensibilidade às condições iniciais, passível de aparecimento uma vez que $F$ e $G$ podem perfeitamente ser nãolineares. Nessas condições, embora haja equações determinísticas modelando o fenômeno, seu comportamento dinâmico torna-se caótico e, portanto, imprevisível (Bai-Lin, 1990).

Outra questão seria tanto física e biológica quanto filosófica e consideraria, em equações como as propostas, a irreversibilidade presente em todos os processos naturais. A questão poderia ser respondida pelo simples fato de as funções $F$ e $G$ poderem ser dissipativas, ou seja, poderem expressar aumento ou diminuição de volume no espaço de estados (Ott, 1993).

Talvez a questão relativa ao fato de o mesmo sistema biológico responder de maneira diferente ao mesmo estímulo, aplicado em momentos diferentes, seja difícil de responder. Acreditamos que a resposta a essa questão fique por conta da sensibilidade às condições iniciais e das bifurcações inerentes a certos processos dinâmicos não-lineares (Ruelle, 1991).

\section{Complexidade, probabilidade, evolução temporal: um pouco de auto-organização}

Nesta seção trataremos de outra condição para que, do ponto de vista biológico, um sistema possa ser considerado complexo. Assumiremos que a condição expressa 
anteriormente, a respeito da heterogeneidade, esteja satisfeita. Isto é, que o sistema biológico complexo seja composto de partes descritas por equações dinâmicas do tipo (2) e esteja agrupado de maneira a seguir uma evolução temporal governada por equações do tipo (3). A condição a ser discutida aqui referir-se-á ao agrupamento das partes, atribuindo a ele um mecanismo que aparentemente não seguirá apenas o acaso.

Retomando o texto de Dawkins, verificamos a existência de diferentes formas de se agrupar as peças de um avião, mas apenas uma, ou uma pequena quantidade delas, resulta efetivamente em um arranjo capaz de satisfazer aquilo que se esperaria de um avião. Ou seja, por trás do agrupamento das peças, há um projeto a ser satisfeito.

Analogamente, há muitas maneiras de se agrupar as diversas partes de um organismo complexo, mas apenas uma, ou uma pequena quantidade delas, resultaria em algo capaz de exercer certas funções naturais como reprodução, alimentação e adaptação ao meio ambiente.

Seria como se um sistema biológico complexo tivesse sido projetado com determinada finalidade e de acordo com certas especificações. Entretanto, tais especificações não foram estabelecidas previamente, o que sugeriria a seleção natural atuando na auto-organização (Darwin, 1859).

Do ponto de vista da formalização da idéia, haveria duas coisas diferentes a serem combinadas: a grande quantidade de arranjos possíveis das partes e uma espécie de avaliação da adequação do arranjo visando uma certa finalidade.

Apesar de termos dúvidas quanto a essas idéias serem matematizáveis, tentaremos fazê-lo partindo do pressuposto de que o sistema complexo, constituído das $m$ partes já citadas e descrito pelas equações (2) e (3), resulta de uma evolução temporal no sentido de aumento de complexidade, ou seja, resulta naturalmente de sistemas mais simples mediante processos de agrupamento e divisão. A esse processo de aumento de complexidade chamaremos de processo de auto-organização do sistema biologicamente complexo.

As idéias que acabamos de expor nos levaram a concluir pela necessidade de serem estabelecidas grandezas que pudessem medir a complexidade de um sistema de maneira objetiva, associando valores numéricos às situações pelas quais passa ao longo de sua história.

A evolução temporal dessas grandezas deve, em princípio, dar uma idéia do mecanismo de organização subjacente e ser aplicável às mais diversas escalas temporais. Assim, será possível estabelecer as variações de complexidade sob os pontos de vista filogenético, ontogenético e da dinâmica de um processo ao longo da vida de um dado sistema.

Considerando as hipóteses anteriores, definiremos inicialmente a grandeza $\left(C_{\mathcal{e}}\right)$, que chamaremos de complexidade estrutural. Essa complexidade $\left(C_{\mathcal{e}}\right)$ será 
definida levando-se em conta a quantidade de partes do sistema e o número de agrupamentos possíveis dessas partes, fazendo uso da idéia de entropia informacional que em alguns casos tem definição análoga à de entropia termodinâmica (DenisPapin \& Cullmann, 1976).

Para levarmos em conta o número de partes do sistema, definiremos a grandeza $C_{1}$, que será denominada complexidade estrutural parcial e dada por:

$$
C_{1}=k \log _{b} m
$$

A constante de proporcionalidade $k \mathrm{e}$ a base da função logarítmica $b$ podem ser escolhidas arbitrariamente e, uma vez selecionadas, definem a unidade de complexidade utilizada.

Por uma questão de comodidade escolheremos $k=1, b=2$ e a complexidade será medida em bits. Isto é, tentaremos medir a complexidade do sistema pelo número de bits que um sistema de computação precisaria memorizar para poder descrevê-lo (Hopf, 1988).

Para completar a definição da medida da complexidade estrutural, conceituaremos um termo que considera os agrupamentos possíveis das $m$ partes e a probabilidade de ocorrência de cada um desses agrupamentos. Para tanto, será definida a grandeza $\eta$, que denominaremos índice de correção da complexidade estrutural.

Consideraremos, então, que as m partes do sistema possam se agrupar de $Q$ maneiras possíveis, sendo a cada uma delas associada uma certa probabilidade $p_{i}$. Nessas condições será possível definir a entropia informacional $\left(H_{g}\right)$ relativa aos agrupamentos pela equação:

$$
\begin{gathered}
Q \\
H_{\mathfrak{g}}=\sum_{i=1} \log _{2}\left(1 / p_{i}\right)
\end{gathered}
$$

Como o máximo valor teórico da entropia informacional relativa aos agrupamentos $\left(H_{g m}\right)$ ocorre quando todos eles são eqüiprováveis (Guiasu \& Theodorescu,1968), ela é dada por:

$$
H_{g m}=\log _{2} Q
$$

A partir dessa equação, definiremos o índice de correção da complexidade estrutural $(\eta)$ pelo quociente entre a entropia informacional relativa aos agrupamentos $\left(H_{\mathfrak{g}}\right)$ e seu máximo valor teórico $\left(H_{\mathfrak{g} m}\right)$. Assim,

$$
\eta=\frac{H_{\mathscr{g}}}{H_{\mathscr{g} m}}
$$


Antes de completar a definição de complexidade estrutural $\left(C_{\mathcal{e}}\right)$ usando as grandezas definidas pelas equações (4) e (7), procuraremos discutir o significado de cada uma delas, enfatizando seus mecanismos de evolução temporal.

A complexidade estrutural parcial $\left(C_{l}\right)$ mede a quantidade de partes relevantes em que o sistema pode ser decomposto. Dos pontos de vista filogenético e ontogenético é uma grandeza que aumenta ao longo do tempo.

O índice de correção da complexidade estrutural $(\eta)$ é expresso por um número real, compreendido no intervalo $[0 ; 1]$ e pode ser utilizado para expressar a adequação do sistema biológico ao meio. Assim, à medida que o número de agrupamentos adequados e possíveis diminui, o valor de $\eta$ diminui, uma vez que as diversas possibilidades deixam de ser eqüiprováveis.

Tal raciocínio permite concluir que quanto mais complexo o sistema, maior o valor de $C_{1}$ e menor o valor de $\eta$, levando-nos a definir complexidade estrutural $\left(C_{\mathcal{e}}\right)$ como:

$$
C_{e}=(I-\eta) C_{1}
$$

Para completar a definição de complexidade, é necessário levar em conta os processos dinâmicos que podem ocorrer no sistema por intermédio das soluções atratoras possíveis. Tais soluções são relacionadas com o regime permanente das equações dinâmicas dadas em (3).

Considerando que o número de soluções atratoras possíveis do sistema seja $P$, a complexidade funcional do sistema $\left(C_{f}\right)$ poderá ser definida por:

$$
C_{f}=\log _{2} P
$$

Agora será possível definir complexidade global do sistema $\left(C_{S}\right)$ pelo produto de suas complexidades estrutural e funcional:

$$
C_{s}=C_{e} \cdot C f
$$

A definição dada pela equação (10) permitirá considerarmos que a grandeza $C_{\mathcal{S}}$ será variável no tempo e, quando seu valor for crescente, o processo evolutivo será chamado de temporalmente auto-organizado.

Encerraremos esta seção ressaltando que alguns pontos importantes das idéias de Dawkins foram discutidos. A idéia de complexidade, associada à grande quantidade de partes envolvidas, foi expressa através da grandeza $C_{1}$. Além disso, a diversidade de arranjos possíveis, associada à aparente intencionalidade do arranjo, foi expressa através da grandeza $\eta$. Os comportamentos possíveis do sistema têm sua multiplicidade expressa na definição da complexidade funcional $\left(C_{f}\right)$. Todos esses fatores, agrupados, estabelecem o conceito de complexidade global do sistema $\left(C_{s}\right)$. O aumento da complexidade global do sistema $\left(C_{s}\right)$ implica organização do sistema nas escalas filogenética, ontogenética e da dinâmica de um dado processo. 


\section{A adequação do sistema biológico}

Outro ponto central no texto de Dawkins refere-se ao sistema biológico complexo ser, de maneira aparentemente proposital, arranjado de tal forma a ser adequado à execução de certas tarefas básicas necessárias à sua sobrevivência e adaptação ao meio.

Nas palavras de Dawkins, o sistema composto de partes, aparentemente ligadas de acordo com um certo projeto, tem essas partes relacionadas. Nessas condições, uma certa complexidade está associada ao sistema de maneira a torná-lo apto a manter sua vida.

Ao longo do tempo, o sistema biológico complexo muda seus mecanismos de agrupamento e seus parâmetros constitutivos, criando para si a capacidade de aplicar certos tipos de forças especiais que, sob certas circunstâncias, podem realizar trabalho, provendo a energia necessária para tarefas como nadar, voar ou andar em busca de alimento e manutenção da vida.

A tentativa de formalizar tal idéia considera como pressuposto básico, que, embora o sistema, em determinado instante seja composto por $\mathrm{m}$ partes e que sua dinâmica seja descrita por equações do tipo (3), o número de partes, parâmetros constitutivos e mecanismos de interligação podem variar ao longo do tempo nas suas mais diversas escalas.

Assim, à medida que o sistema se ajusta ao meio ao longo do tempo, os valores das grandezas $C_{l}, \eta, C_{e}, C_{f}$ e $C_{s}$ assumem valores variáveis dos pontos de vista filogenético, ontogenético e da dinâmica local de um dado processo.

Do ponto de vista filogenético, as comparações devem ser feitas entre indivíduos de diversas espécies, tomados em situações temporais diferentes. A adequação do sistema ao meio, viabilizando a vida, talvez seja feita com valores de $C_{l}, C_{e}, C_{f}$ e $C_{\boldsymbol{s}}$, que podem ser crescentes ou decrescentes, pois o número de partes e o número de atratores possíveis pode aumentar ou diminuir à medida que o sistema melhora seus mecanismos de adequação ao meio. $\mathrm{O}$ índice de correção da complexidade estrutural $(\eta)$, entretanto, diminui uma vez que nessas condições alguns arranjos ficam cada vez mais prováveis do que os outros.

Do ponto de vista ontogenético, as comparações devem ser feitas entre situações temporais diferentes de um mesmo indivíduo, que é observado em conjunto com seus diversos processos ao longo da vida.

Considerando agora um certo processo dinâmico, ao compará-lo instante por instante, a variável $C_{f}$ é a mais relevante. Tal relevância se deve ao fato de essa variável relacionar-se com os atratores do sistema, fundamentais para a descrição do comportamento particular dos processos que têm lugar em determinado indivíduo.

Todas as considerações feitas até aqui, embora de interesse, referem-se apenas às medidas comparativas entre sistemas ao longo de processos de adequação ao 
meio ambiente. Falta ainda expressar o que significa, do ponto de vista formal, o processo de adequação de um sistema ao seu meio.

Talvez o mecanismo de adequação possa ser expresso, nas escalas filogenética e ontogenética, pelo conceito de estabilidade estrutural. Na dinâmica de processos particulares que ocorrem em um certo indivíduo, a adequação ambiental pode ser expressa definindo-se uma função do vetor das variáveis de estado $X_{i}$, de maneira semelhante às funções de Liapunov, utilizadas para expressar a estabilidade de soluções atratoras em sistemas dinâmicos (Guckenheimer \& Holmes, 1983).

Para maior clareza, recorreremos a outro exemplo citado por Dawkins. Apesar de vivermos em ambientes com as mais variadas temperaturas, nosso sistema termoregulador mantém a temperatura do organismo em um valor de aproximadamente $36,5^{\circ} \mathrm{C}$. Isso se dá por meio de mecanismos internos de conversão de energia química em mecânica e térmica que proporcionam condições suficientes à manutenção da temperatura ideal para a ocorrência de reações químicas, caracterizando processos de controle e regulação retroalimentados (Segel, 1984).

Assim, consideraremos que um processo dinâmico particular que ocorre em um certo indivíduo, cuja dinâmica será descrita por equações do tipo (3) admitindo soluções atratoras em torno das quais será possível definir funções de Liapunov do tipo $V\left(X_{i}\right)$.

A escolha das funções de Liapunov é em geral fundamentada no conceito de energia do sistema e sua relação de dependência com as variáveis de estado expressa a tendência à estabilidade das soluções atratoras. Essas funções, em vizinhanças abertas das soluções atratoras, devem seguir as seguintes condições:

- $V\left(X_{i}\right)=0$ para todo $X_{i}$ pertencente à solução atratora.

- $V\left(X_{i}\right)>0$ para todo $X_{i}$ fora da solução atratora.

- $\dot{V}\left(X_{i}\right)<0$ para todo $X_{i}$ nas vizinhanças da solução atratora.

Tais condições indicam que as soluções atratoras, correspondentes aos processos dinâmicos que ocorrem em sistemas complexos, são assintoticamente estáveis (Guckenheimer \& Holmes, 1983). O raciocínio descrito significa que os organismos, operando em condições normais, possuem dinâmica determinada por atratores no espaço de estados correspondentes a condições homeostáticas.

Condições patológicas correspondem a mudanças nos parâmetros $\Gamma_{i}$ da equação do tipo (3), que descrevem o comportamento do sistema nessa escala temporal. A mudança de parâmetros $\Gamma_{i}$ traz como conseqüência mudanças qualitativas nos atratores do sistema. Os novos atratores assim formados deixam o sistema fora das condições energéticas necessárias para que os processos de controle e regulação ocorram, e requerem para seu restabelecimento que os parâmetros $\Gamma_{i}$ voltem aos valores habituais por mecanismos endógenos e exógenos. 
A ontogênese desses processos poderia ser considerada supondo-se também equações do tipo (3), cujos parâmetros $\Gamma_{i}$ correspondentes aos mecanismos fisiológicos do indivíduo, variam ao longo da vida. Essas variações podem ser acompanhadas por mudanças qualitativas, nos processos descritos, correspondendo a bifurcações (Murray, 1993).

Do ponto de vista matemático, tais bifurcações correspondem às situações de instabilidade estrutural; do ponto de vista biológico, à organização ontogenética do sistema complexo.

Exemplificando: um bebê, ao nascer, possui uma grande variedade de ritmos ultradianos na composição do espectro de seu ciclo vigília-sono. À medida que o tempo vai passando, esses ritmos vão diminuindo de importância, estabelecendo-se como mais significativo o circadiano. Essas mudanças qualitativas correspondem a passagens de alguns parâmetros neuro-biológicos por valores de bifurcação, alterando qualitativamente a dinâmica do ciclo vigília-sono (Benedito-Silva, 1993).

$\mathrm{Na}$ escala filogenética seria, talvez, possível a associação de mudanças qualitativas ao longo das espécies, mediante bifurcações globais correspondentes a mudanças na forma das expressões de $G_{i}$, representativas dos mecanismos dinâmicos gerais das equações do tipo (3), utilizadas na modelagem dos processos biológicos complexos.

Assim, processos de controle e regulação descritos por equações do tipo (3) e restritos a certa funções de Liapunov, resultam de adequação de parâmetros $\Gamma_{i}$ ao longo da vida. A forma das equações, entretanto, resulta da filogênese dos mecanismos de adequação do sistema complexo às condições ambientais.

\section{A ontogênese do ciclo vigília-sono}

As idéias teóricas aqui apresentadas podem ser utilizadas em problemas biológicos, conforme exemplificaremos a seguir, utilizando dados a respeito do estabelecimento do ritmo circadiano do ciclo vigília-sono (CVS) no início da vida.

A alternância entre o sono e a vigília é um ritmo biológico cujo padrão temporal é caracterizado pela freqüência dessas alternâncias. No CVS humano podem ser detectadas simultaneamente freqüências ultradianas, correspondentes a períodos menores que 20 horas; circadianas, correspondentes a períodos maiores que $20 \mathrm{e}$ menores que 28 horas; infradianas, correspondentes a períodos maiores que 28 horas (Halberg et al., 1977).

O padrão temporal do CVS modifica-se ao longo da ontogênese, e essas modificações apresentam variações interindividuais importantes.

No recém-nascido, a alternância da vigília com o sono é pouco estável, com horários incertos de início e fim, que tendem a assumir gradativamente uma organização circadiana, inicialmente desvinculada de fatores ambientais. A partir de certa idade, o CVS passa a apresentar sincronização com os ciclos ambientais, 
isto é, a criança passa a dormir um sono longo à noite, fazendo uma sesta pela manhã e outra à tarde que tendem a desaparecer com o passar do tempo.

Esse padrão persiste durante a infância e adolescência, etapa do desenvolvimento em que o CVS continua com um padrão circadiano estável, mas os inícios de sono ficam mais incertos e passam a ocorrer mais tarde (Carskadon et al., 1993).

Durante a fase adulta o componente circadiano predomina, mantendo-se estável e previsível, tendendo a se atenuar na velhice com o aparecimento dos cochilos (Bliwise, 1993).

Parece-nos, então, razoável descrever o desenvolvimento do CVS por meio da medida de entropia de agrupamento $\left(H_{g}\right)$ dada em $(5)$, considerando como partes possíveis os diversos componentes do espectro de freqüências.

Assim, o CVS seria inicialmente considerado instável e imprevisível, pois vários componentes do espectro de freqüências podem aparecer, caracterizando uma certa uniformidade na distribuição de probabilidades. Com o passar do tempo o CVS migra para uma condição mais estável e previsível com a predominância do componente circadiano, desbalanceando a distribuição de probabilidades. Na terceira idade parece haver uma retomada parcial da condição inicial de instabilidade e baixa previsibilidade, pois o componente circadiano se atenua e os ultradianos ganham força relativa com o aparecimento dos cochilos.

Dados de CVS de recém-nascidos serão utilizados neste trabalho com o intuito de mostrar a aplicação da medida de entropia de agrupamento como indicativa do grau de complexidade do sistema de temporização ao longo do processo de estabelecimento do padrão circadiano.

Fundamentalmente apresentaremos uma maneira de calcular o parâmetro relacionado com a entropia de agrupamento $\left(H_{g}\right)$, mostrando que sua evolução temporal pode medir o grau de complexidade e organização do sistema de temporização representado pelo ciclo vigília-sono em função do tempo. Assim, seria esperado que $H_{g}$ diminuísse, implicando a diminuição de $\eta$. Mantidas as outras condições relativas ao processo, teremos caracterizado um aumento de complexidade e uma conseqüente auto-organização.

Cinco bebês, três do sexo feminino (F1, F2 e F3) e dois do sexo masculino (M1 e M2), nascidos a termo e saudáveis, foram selecionados dentre famílias conhecidas (1). Foi solicitado aos pais que observassem e anotassem todos os horários de início e fim de sono desses bebês durante os seus três primeiros meses de vida.

Agrupados em blocos de uma semana, esses dados foram utilizados no cálculo das durações dos episódios de sono e de vigília para cada criança separadamente. Tais durações foram divididas em intervalos de 10 minutos, com o intuito de se construir séries temporais com dados eqüidistantes.

Dessa forma, o CVS de cada criança foi semanalmente descrito por uma série temporal de sete dias, na qual cada ponto representa um estado entre dois possíveis: 
ou a criança está dormindo ou está acordada. As séries temporais do CVS das cinco crianças, separadas em blocos de uma semana, foram submetidas à transformada rápida de Fourier. Assim, produziram um periodograma para cada semana de vida de cada criança.

Os componentes espectrais presentes nos periodogramas foram considerados no cálculo da medida de entropia de agrupamento Hg. Em cada periodograma, cada componente espectral foi tomado como uma mensagem que continha certa quantidade de informação relacionada à freqüência relativa de ocorrência.

A partir da quantidade de informação contida em cada componente espectral, calculamos a medida de entropia de agrupamento correspondente à quantidade de informação total contida no periodograma (2). Na figura 1 , mostramos a evolução da entropia de agrupamento $\left(H_{\mathfrak{g}}\right)$, medida em bits, em função da idade da criança, em semanas.

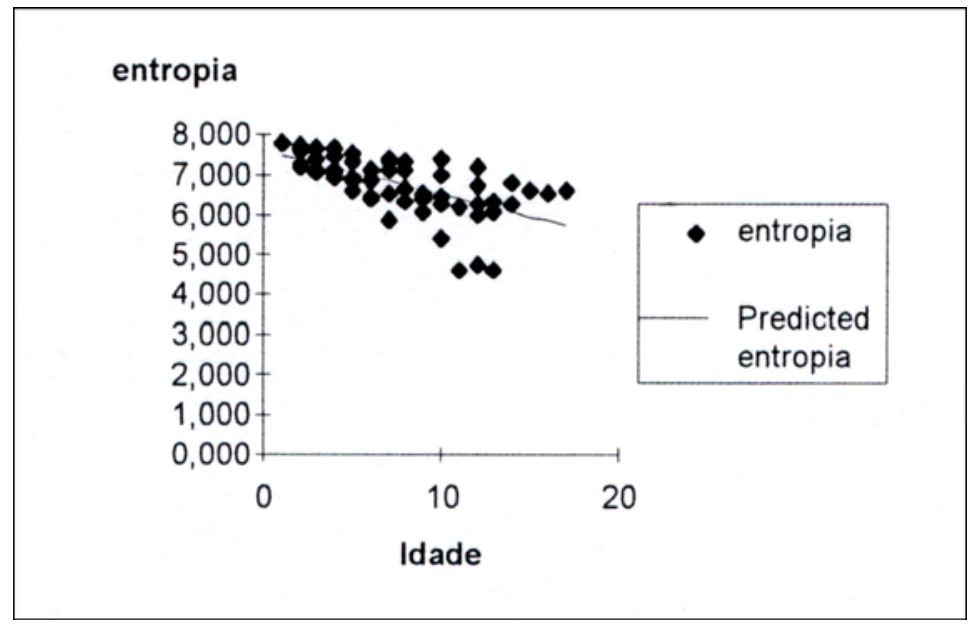

Figura 1: Entropia versus idade

Como pode ser observado, a medida de entropia diminui com a idade, caracterizando um aumento na complexidade do CVS ao longo do tempo. Apesar disso, a taxa dessa diminuição varia dependendo da criança e, conseqüentemente, para uma mesma idade as crianças apresentam muitas vezes $\mathrm{Hg}$ diferentes.

Do ponto de vista biológico é interessante notar ser o resultado obtido coerente: indica auto-organização no CVS das diversas crianças e ressalta as diferenças individuais expressas pelos diferentes valores de $\mathrm{Hg}$ para as diferentes crianças em uma certa idade. 


\section{Conclusões}

Tentamos relacionar as idéias de complexidade expostas por Dawkins com conceitos matemáticos originários da Teoria da Informação e da Teoria dos Sistemas Dinâmicos, procurando contribuir para uma modelagem não limitada à análise de sinais.

A procura de conceitos visa estabelecer algum corpo formal aos problemas biológicos, adaptável aos seus diversos tipos nas diversas escalas temporais. $\mathrm{O}$ estudo apresentado procurou mostrar um caso de aplicação a dados biológicos, descrevendo fenômenos qualitativamente observáveis de maneira quantitativa.

Várias questões deixaram de ser discutidas como, por exemplo, a dos níveis de organização, perfeitamente compatíveis com o enfoque desse artigo e que, talvez, possam ser objeto de estudo e de trabalhos futuros.

Notas

1 Estes dados fazem parte do projeto de Iniciação Científica de Clarissa Bueno, sob orientação do Prof. Dr. Luiz Menna-Barreto, que autorizou a sua publicação.

2 Os cálculos detalhados fazem parte da tese de doutorado de Ana Amélia BeneditoSilva, apresentada na EP-USP.

Referências bibliográficas

BAI-LIN, H. Chaos II. Singapure, World Scientific Publishing Co., 1990.

BENEDITO-SILVA, A..A. Ontogênese do ciclo vigília-sono em bebês. São Paulo, 1993. Dissertação (Mestrado). Escola Politécnica da Universidade de São Paulo.

BERTALANFFY, L. von. General systems theory. New York, George Braziller, Inc., 1968.

BLIWISE, D.L. Sleep in normal aging and dementia. Sleep, v.16, p. 40-81, 1993.

CARSKADON, M.A.; VIEIRA, C. \& ACEBO, C. Association between puberty and delayed phase reference. Sleep, v.16, p. 258-262, 1993.

COVENEY, P. \& HIGHFIELD, R. Frontiers of complexity. New York, Fawcett Columbine, 1995.

DARWIN, C. The origin of species. USA, Mentor Book Co., 1958 [reprinted from 1859].

The descent of man. New Jersey, Princeton University Press, 1981 [reprinted from 1871].

DAWKINS, R. The blind watchmaker. London, Penguin Books, 1986. 
DENIS-PAPIN, M. \& CULLMANN, G. Elementos de cálculo informacional. Bilbao, Urmo S.A.Ediciones, 1976.

GOULD, S.J. Ontogeny and phylogeny. Massachusetts, The Belknap Press of Harvard University Press, 1977.

GUCKENHEIMER, J. \& HOLMES, P. Nonlinear oscillations, dynamical systems, and bifurcation of pector fields. New York, Springer Verlag, 1983.

GUIASU, S. \& THEODORESCU, R. La théorie mathématique de l'information. Paris, Dunod, 1968.

HALBERG, F.; CARADENTE, F.; CORNÉLISSEN, G. \& KATINAS, G.S. Glossary of chronobiology. Chronobiologia, v.4, suppl.1, p.189, 1977.

HOPF, F.A. Entropy and evolution. In: Entropy, information and evolution. Cambridge, Massachusetts, MIT Press, 1988.

LOÈVE, M. Probability theory. Berlin, Springer Verlag, 1963.

MAINZER, K. Thinking in complexity. Berlin, Springer Verlag, 1994.

MURRAY, J.D. Mathematical biology, 2nd. Ed. Berlin, Springer Verlag, 1993.

OTT, E. Chaos in dynamical systems. Massachusetts, Cambridge University Press, 1993.

PIQUEIRA, J.R.C. Estabilidade estrutural e organização. Coleção Documentos. São Paulo, IEA-USP, jul. 1992.

Structural and functional complexity: an informational approach.

IEEE Systems, Man and Cybernetics, San Antonio, Texas, p. 1974-1978, Oct. 1994.

RUELLE, D. Chance and chaos. Oxford, Princeton University Press, 1991.

SEGEL, L.A. Modeling dynamic phenomena in molecular and cellular biology. Massachusetts, Cambridge University Press, 1984.

SHANNON, C. \& WEAVER, W. The mathematical theory of communication, 12th printing. Chicago, University of Illinois Press, 1971 [reprinted from 1949].

SHUMWAY, R.H. Applied statistical time series analysis. New Jersey, Englewood Cliffs, Prentice-Hall, 1988.

RESUMO - NESTE ARTIGO tenta-se estabelecer algumas interpretações teóricas para os conceitos de auto-organização e complexidade em sistemas biológicos, utilizando-se idéias originárias da Teoria da Informação e da Teoria dos Sistemas Dinâmicos. Além disso, apresenta-se um exemplo de aplicação dos métodos propostos ao problema da caracterização do grau de organização do ciclo vigília-sono em bebês.

ABSTRACT - THIS ARTICLE is a trial on stablishing some theoretical formalisms for the concepts of self-organizing and complex biological systems, using ideas from Information Theory and Dynamical Systems Theory. As an example the organization of the sleep-wake cycle in babies is studied, applying the methods proposed. 
Os autores agradecem ao Grupo Multidisciplinar de Ritmos Biológicos do ICB-USP, ao CLE-HC da Unicamp e ao LAC da EP-USP. Em especial, ao IEA-USP pela oportunidade de desenvolverem os seminários de Biomatemática, fundamentais para a elaboração do artigo.

José Roberto Castilho Piqueira é professor da Escola Politécnica da Universidade de São Paulo. E-mail: piqueira@lac.usp.br

Ana Amélia Benedito-Silva é pesquisadora da Escola Paulista de Medicina da Universidade Federal de São Paulo. 